\title{
Orthotropic Non-hysteretic Permeability Model as a Lookup-table for FEA
}

\author{
Robert Courant* , Jürgen Maas \\ Mechatronic Systems Laboratory, Technische Universität Berlin, 10623 Berlin, Germany \\ *robert.courant@emk.tu-berlin.de
}

\begin{abstract}
SNE 31(2), 2021, 65-72, DOI: 10.11128/sne.31.tn.10563
Received: May 23, 2021 (Selected ASIM SST 2020 Postconf.

Publ.), Accepted: May 31, 2021

SNE - Simulation Notes Europe, ARGESIM Publisher Vienna, ISSN Print 2305-9974, Online 2306-0271, www.sne-journal.org
\end{abstract}

Abstract. Within this paper, we present an appropriate approach to prepare the data of orthotropic nonlinear soft magnetic materials for use in finite element analyses. Based on the co-energy density, an elliptic model for the interpolation between the principal directions is derived and extended to laminated materials. Instead of adapting the FE-code to incorporate the fundamental equations, a workflow is proposed to generate a lookup-table of the anisotropic nonlinear permeability which can be easily integrated in FE-programs like COMSOL Multiphysics. The modelled behaviour is shown for typical material classes and the lamination model is numerically validated.

\section{Introduction}

Soft magnetic materials are essential for almost all magnetic circuits. They play an important role in electromotors and transformers to guide the flux to guarantee an efficient operation[1],[2]. For the dimensioning of those circuits, accurate models of their magnetic behaviour are necessary. The most important characteristic is the relation between magnetic field strength $\mathbf{H}$ and magnetic flux density B. All ferromagnetic materials show a saturation at high fields, therefore a nonlinear approach has to be chosen. As the nonlinear problems are almost impossible to solve analytically for complex geometries, magnetic problems are typically computed numerically.

High performance soft magnetic materials often exhibit an anisotropic behaviour. Characteristic examples are different grain oriented (GO) electrical steels that show a magnetic easy axis in rolling direction and transverse magnetic hard axes. Consequently, magnetically anisotropic materials are typically characterised by uniaxial nonlinear curves $B_{\mathrm{u}, i}\left(H_{\mathrm{u}, i}\right)$ in three principal directions. This experimental characterisation of anisotropic materials requires sophisticated measurements[3],[4]. Because the flux components influence each other (even for isotropic materials), for vectors between the principal directions, a suitable interpolation has to be found. An extension of the topic are laminated materials, where non-ferromagnetic layers inhibit the perpendicular flux more than in plane.

This paper chooses an elliptical approach based on the co-energy density, which is well known concept[5],[6]. Key elements are based on the implementation of [7]. It has to be noted that hysteresis is not considered here. For magnetic materials, hysteresis is typically modelled with a JILES-ATHERTON model, other models like PREISACH or PRANDTL-ISHLINSKI are also applicable.

Another application of anisotropic magnetic properties, that attracted the author to the topic in the first place, are magnetic shape memory alloys, studied for their shape memory and magnetocaloric effect. These are indeed described by BH-curves in easy direction and in the perpendicular hard directions as shown in Figure 1. Therefore, they are appropriate benchmarking examples for our model.

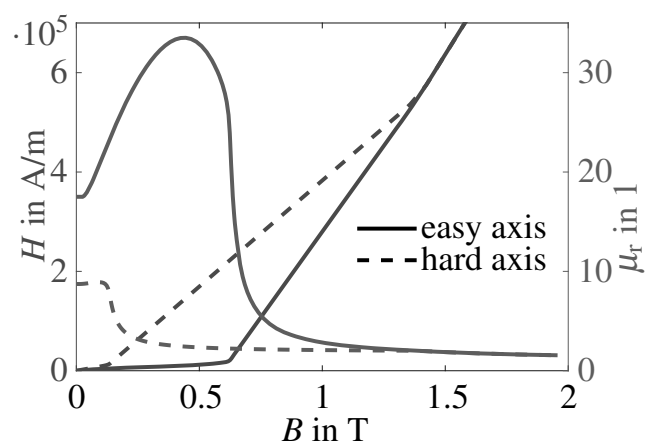

Figure 1: HB- and $\mu_{\mathrm{r}}(B)$-curves for easy and hard axis of examined MSMA from [8].

Section 1 introduces the physical background and motivates the elliptical model. This approach is gener- 
alized for nonlinear orthotropic materials in section 2 . Section 3 extends the model to laminated materials. Because magnetic anisotropy can be only incorporated as a relative permeability in the used FE-software COMSOL Multiphysics, the model is prepared as a look-up table of the nonlinear permeability dependent on the flux density B. In section 4, our model is analysed for different example materials. The laminate is numerically validated in section 5.

\section{Constitutive Equations}

Electromagnetic problems are described by MAXWELL's equations. There, two quantities each are defined for the electric and magnetic domain, one for the excitation and one for the effect. In the magnetic domain, these are the magnetic field strength $\mathbf{H}$ and the magnetic flux density $\mathbf{B}$. These two vector fields are coupled by generally nonlinear material properties

$$
\mathbf{B}=\mathbf{B}(\mathbf{H}) .
$$

The co-energy density of the magnetic field can be derived by

$$
w^{\prime}(\mathbf{B})=\int_{0}^{\mathbf{B}} \mathbf{H} \cdot \mathrm{d} \tilde{\mathbf{B}} .
$$

In the easiest case of vacuum this is

$$
w^{\prime}(\mathbf{B})=\frac{\mathbf{B}^{2}}{2 \mu_{0}}
$$

which leads to the linear coupling of $\mathbf{B}$ and $\mathbf{H}$

$$
\mathbf{B}=\mu_{0} \mathbf{H} .
$$

For general linear materials, this is extended using Einstein notation to

$$
B_{j}=\mu_{0} \mu_{\mathrm{r}, i j} H_{i}
$$

with the relative permeability matrix $\mu_{\mathrm{r}, i j}$. In most cases, the relative permeability is assumed orthotropic and the matrix is thus

$$
\frac{1}{\mu_{\mathrm{r}, i j}}=\left(\begin{array}{ccc}
\frac{1}{\mu_{\mathrm{r}, x}} & 0 & 0 \\
0 & \frac{1}{\mu_{\mathrm{r}, y}} & 0 \\
0 & 0 & \frac{1}{\mu_{\mathrm{r}, z}}
\end{array}\right) .
$$
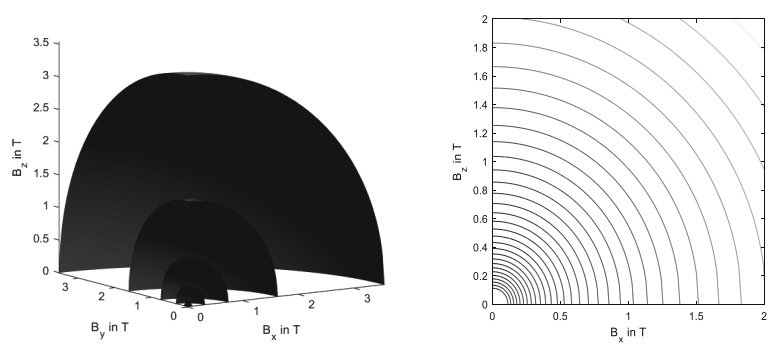

Figure 2: Contour lines of the magnetic co-energy for a linear isotropic material with $\mu_{\mathrm{r}, \text { iso }}=5000$.
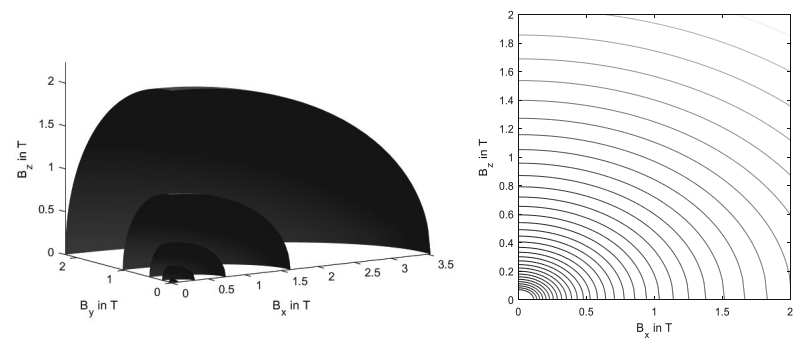

Figure 3: Contour lines of the magnetic co-energy for a linear anisotropic material.

The co-energy density for this case is

$$
\begin{aligned}
w^{\prime}(\mathbf{B}) & =\int_{0}^{\mathbf{B}} \frac{1}{\mu_{0} \mu_{\mathrm{r}, i j}} \tilde{B}_{j} \cdot \mathrm{d} \tilde{B}_{i} \\
& =\frac{B_{x}^{2}}{2 \mu_{0} \mu_{\mathrm{r}, x}}+\frac{B_{y}^{2}}{2 \mu_{0} \mu_{\mathrm{r}, y}}+\frac{B_{z}^{2}}{2 \mu_{0} \mu_{\mathrm{r}, z}} .
\end{aligned}
$$

The contour surfaces $w^{\prime}=$ const. of this are concentric ellipsoids. This is why an elliptic model is used in section 4 to model anisotropic nonlinear functions. Figure 2 depicts the contour surfaces in $3 \mathrm{D}$ or contour lines in $2 \mathrm{D}$ for a linear isotropic material with $\mu_{\mathrm{r} \text {,iso }}=5000$. The contour surfaces are concentric spheres. Figure 3 shows the expected ellipsoids for a linear anisotropic material with $\mu_{\mathrm{r}, x}=5000$ and $\mu_{\mathrm{r}, y}=\mu_{\mathrm{r}, z}=2000$.

Nonlinear isotropic materials have equally oriented field and flux, where the absolute value is described by a nonlinear relation as

$$
\mathbf{B}=B_{\text {iso }}(|\mathbf{H}|) \frac{\mathbf{H}}{|\mathbf{H}|} .
$$

The use of the absolute value produces a spherical interpolation between the directions. Although the shown relation $\mathbf{B}(\mathbf{H})$ is the more comprehensible, for numerical 
validations the opposite formulation $\mathbf{H}(\mathbf{B})$ is preferable, because in most FE-programs, the problem is solved using the auxiliary vector potential $\mathbf{A}$ that is linked to the flux density by $\mathbf{B}=\operatorname{rot}(\mathbf{A})$. Accordingly, in section 3 we will setup the lookup-table for the nonlinear function of $\mu_{\mathrm{r}}$ dependent on $\mathbf{B}$ in the form of

$$
\left(B_{x}\left|B_{y}\right| B_{z}\left|\mu_{\mathrm{r}, x}\right| \mu_{\mathrm{r}, y} \mid \mu_{\mathrm{r}, z}\right) .
$$

\section{Elliptic Interpolation}

The interpolation for nonlinear orthotropic materials has to fulfil three conditions. Firstly, it has to be a convex function, which translates to convex contour lines in $2 \mathrm{D}$ or surfaces in $3 \mathrm{D}$. Secondly, the interpolation has to match the uniaxial nonlinear curves it is fitted to. Finally, the approach has to match the elliptical model of the co-energy density as shown in (7) for linear orthotropic magnetic materials. An obvious choice, that meets the requirements, is again an elliptical model of the co-energy density $w^{\prime}(\mathbf{B})$, where the half-axes in all directions of the contour surface for each co-energy density $w^{\prime}$ are determined by the corresponding flux density $B_{\mathrm{u}, i}\left(w^{\prime}\right)$ of the uniaxial measurements.

These measurements are typically provided as a table $\left(H_{\mathrm{u}, i} \mid B_{\mathrm{u}, i}\right)$. The co-energy densities can be numerically integrated according to (2). From the extended table $\left(H_{\mathrm{u}, i}\left|B_{\mathrm{u}, i}\right| w^{\prime}\right)$ a function $B_{\mathrm{u}, i}\left(w^{\prime}\right)$ can be interpolated. For each $w^{\prime}$, the contour surface is described by

$$
\left(\frac{B_{x}}{B_{\mathrm{u}, x}\left(w^{\prime}\right)}\right)^{2}+\left(\frac{B_{y}}{B_{\mathrm{u}, y}\left(w^{\prime}\right)}\right)^{2}+\left(\frac{B_{z}}{B_{\mathrm{u}, z}\left(w^{\prime}\right)}\right)^{2}=1 .
$$

Figure 4 illustrates the two-dimensional case with a contour line. The interpolation scheme is to generate preferably equally spaced sample points of the ellipsoids for each co-energy density $\left(B_{x}\left|B_{y}\right| B_{z} \mid w^{\prime}\right)$. These values can be interpolated to a function $w^{\prime}\left(B_{x}, B_{y}, B_{z}\right)$.

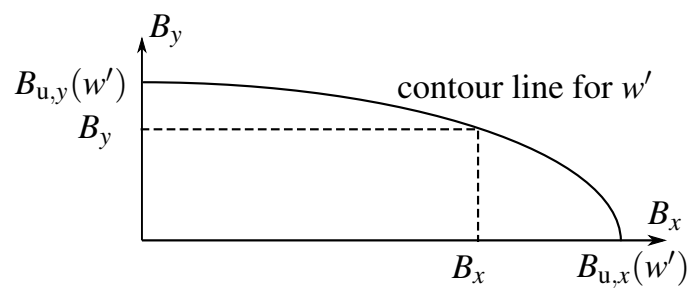

Figure 4: 2D representation of the elliptic interpolation.

\section{Lamination}

Laminated magnetic materials could be modelled with their full geometry as shown in section 5. Due to the thin layers, especially of the non-magnetic insulation, an otherwise unnecessarily fine mesh is required which increases the computational burden. Therefore, for most applications a homogenised material model suffices. The insulation material 2 is non-ferromagnetic $\mu_{\mathrm{r}}=1$. The stacking factor $f$ is the volume ration of the ferromagnetic material 1 to the overall volume. The sheets are thin, a change in the field from one sheet to the next can be neglected. Without loss of generality, the sheets are assumed to be in the xy-plane and stacked in $\mathrm{z}$ direction. At interfaces, the normal component of the flux density is continuous, therefore the homogenized flux density is

$$
B_{z}=B_{1, z}=B_{2, z}
$$

The homogenized flux density in the xy-plane is constructed by a mixing law

$$
\begin{aligned}
& B_{x}=f B_{1, x}+(1-f) B_{2, x} \\
& B_{y}=f B_{1, y}+(1-f) B_{2, y} .
\end{aligned}
$$

The homogenized co-energy density $w_{\text {lam }}^{\prime}$ can be calculated as a weighted sum of that of the ferromagnetic material $w_{1}^{\prime}\left(B_{1, x}, B_{1, y}, B_{1, z}\right)$ from the interpolation and that of vacuum from (3) and is dependent on the homogenized flux density and that in the insulation

$$
\begin{aligned}
& w_{\text {lam }}^{\prime}\left(B_{x}, B_{y}, B_{z}, B_{2, x}, B_{2, y}\right) \\
& =f w_{1}^{\prime}\left(\frac{1}{f}\left(B_{x}-(1-f) B_{2, x}\right), \frac{1}{f}\left(B_{y}-(1-f) B_{2, y}\right), B_{z}\right) \\
& +\frac{1-f}{2 \mu_{0}}\left(B_{2, x}^{2}+B_{2, y}^{2}+B_{z}^{2}\right) .
\end{aligned}
$$

For a strong ferromagnetic material $1, B_{2, x}$ and $B_{2, y}$ can be neglected and the approximated co-energy density is

$$
w_{\text {lam }}^{\prime}(\mathbf{B})=f w_{1}^{\prime}\left(\frac{B_{x}}{f}, \frac{B_{y}}{f}, B_{z}\right)+\frac{1-f}{2 \mu_{0}} B_{z}^{2} .
$$

Relative permeability. The relative permeabilities for the look-up table (9) could be calculated using (5) with the field strength $\mathbf{H}$ derived according to (2) as

$$
\mathbf{H}_{\mathrm{lam}}(\mathbf{B})=\frac{\partial w_{\mathrm{lam}}^{\prime}(\mathbf{B})}{\partial \mathbf{B}} .
$$


While this is physically correct, the numerical derivate on the interpolated function $w_{\text {lam }}^{\prime}(\mathbf{B})$ is not very accurate for a realistic sampling grid. Instead, the permeability can be computed from the uniaxial measurements $\left(H_{\mathrm{u}, i} \mid B_{\mathrm{u}, i}\right)$. Using the analytical derivate of (14), the uniaxial field strengths of the complete laminated material can be calculated as

$$
\begin{aligned}
& H_{\mathrm{lam}, \mathrm{u}, x}\left(B_{\mathrm{u}, x}\right)=H_{\mathrm{u}, x}\left(\frac{B_{\mathrm{u}, x}}{f}\right) \\
& H_{\mathrm{lam}, \mathrm{u}, y}\left(B_{\mathrm{u}, y}\right)=H_{\mathrm{u}, y}\left(\frac{B_{\mathrm{u}, y}}{f}\right) \\
& H_{\mathrm{lam}, \mathrm{u}, z}\left(B_{\mathrm{u}, z}\right)=f H_{\mathrm{u}, z}\left(B_{\mathrm{u}, z}\right)+\frac{1-f}{\mu_{0}} B_{\mathrm{u}, z} .
\end{aligned}
$$

Now, (5) can be solved for $\mu_{\mathrm{r}, \mathrm{lam}, i}\left(B_{\mathrm{u}, z}\right)$. For the uniaxial values, the ferromagnetic material's contribution to the co-energy density

$$
w_{\text {an }}^{\prime}=w_{1}^{\prime}\left(\frac{B_{x}}{f}, \frac{B_{y}}{f}, B_{z}\right)
$$

can be accurately integrated according to (2). While the overall behaviour of the laminate is dependent on the complete co-energy density $w_{\text {lam }}^{\prime}$, the relative permeabilities of the ferromagnetic material are only dependent on the new co-energy density $w_{\text {an }}^{\prime}$. Therefore, the permeabilities of the ferromagnetic material and consequently of the laminate can be derived from the uniaxial measurements. Consequently, the generation of the look-up table (9) consists of two steps: First, $w_{\text {an }}^{\prime}\left(B_{x}, B_{y}, B_{z}\right)$ is calculated on a grid $\left(B_{x}\left|B_{y}\right| B_{z}\right)$, then the permeabilities $\left(\mu_{\mathrm{r}, \mathrm{lam}, x}\left|\mu_{\mathrm{r}, \mathrm{lam}, y}\right| \mu_{\mathrm{r}, \mathrm{lam}, z}\right)$ are taken from the uniaxial measurement with the corresponding co-energy density $w_{\mathrm{an}}^{\prime}$. The presented approach yields a far smoother permeability than the direct derivation according to (15), especially at low flux densities.

\section{Application}

Applying our method to the example depicted in Figure 3 shows no difference. Therefore, the third condition is fulfilled. The second condition can be tested by comparing $\mu_{\mathrm{r}}$ along an axis from the elliptic model with the uniaxial input data. The interpolation shall be applied to a representative GO electrical steel of grade M-6. The $\mathrm{BH}$-curve is taken from the COMSOL material library, the results are shown in Figure 5. The rolling direction has a much higher initial permeability, proportional to the derivate of the curve, than the transverse direction.

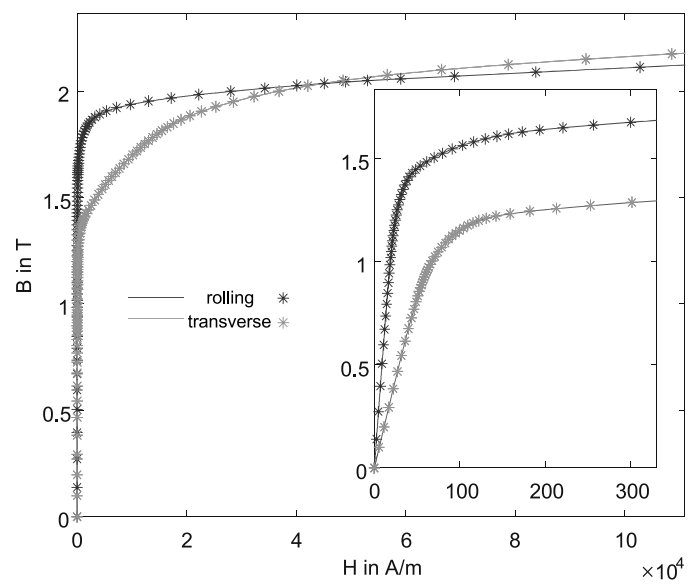

Figure 5: $\mathrm{BH}$-curve of the investigated grain oriented steel M-6 in both principal directions, detail on the right.
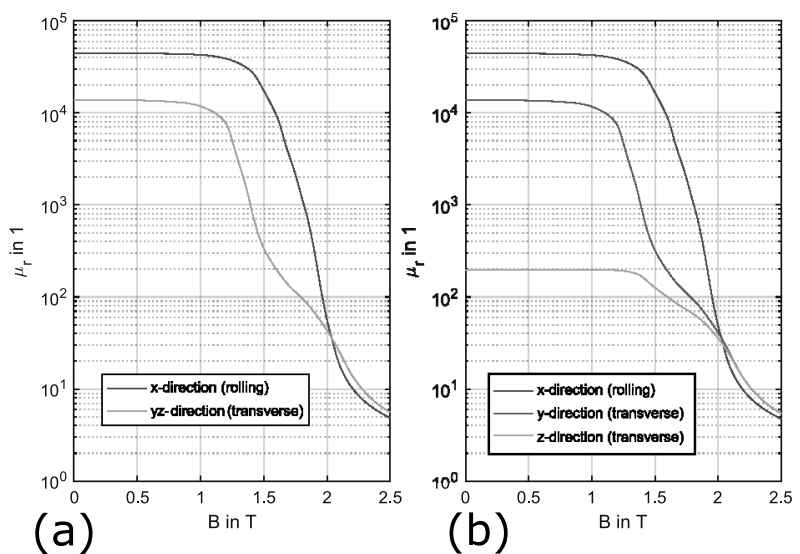

Figure 6: $\mu_{\mathrm{r}, i}\left(B_{\mathrm{r}, i}\right)$ in all directions, (a) of the investigated GO steel M-6 and (b) of the laminate with stacking factor $f=0.995$.

At very high fields, the transverse direction gains a slight advantage. This can also be seen in Figure 6 (a) of the elliptic model for this material with the rolling direction in $x$ and the transverse curves used for $y$ and $z$. The curves of the uniaxial measurements perfectly match the model, therefore the second condition is fulfilled.

The contour surfaces or contour lines in a $2 \mathrm{D}$-section for the elliptical model of this material can be seen in Figure 7. At lower fields, the difference between the axes is higher, almost reaching a factor of two, while the results approximately match at higher fields in accordance with the input curves. The density of the contour lines in the $2 \mathrm{D}$-section is correlated with the permeability. At low fields, the permeability is high and the energy doesn't 

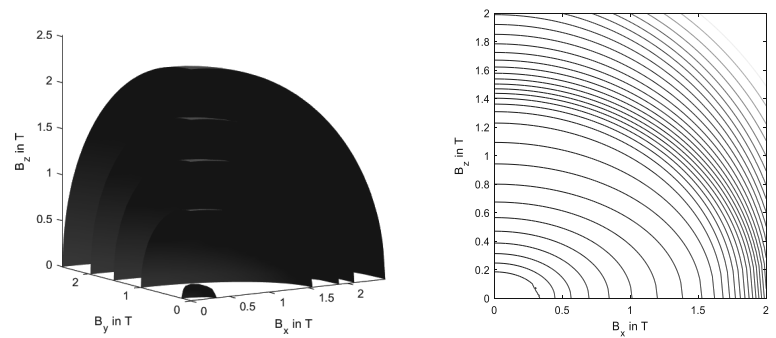

Figure 7: Contour lines of the magnetic co-energy density for pure grain oriented steel without lamination.
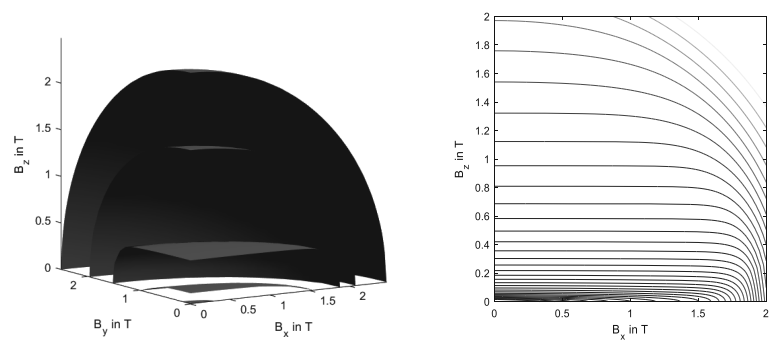

Figure 8: Contour lines of the magnetic co-energy density for laminated grain oriented steel with $f=0.995$.

grow that fast, after the saturation, more energy is needed to still increase the field. When the axes roughly match for high fields, the contour lines are almost concentric circles.

In Figure 8 and Figure 9, laminates of the same grain oriented steel with different stacking factors are shown. In Figure 8, the stacking factor is $f=0.995$, meaning that only $0.5 \%$ are non-magnetic insulation. Nevertheless, for low co-energy densities the flux in z-direction changed drastically. The former ellipses in 2D and ellipsoids in 3D are flattened with an almost parallel top.
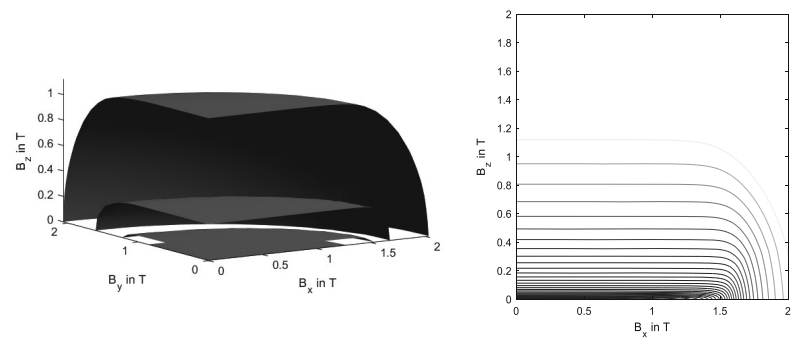

Figure 9: Contour lines of the magnetic co-energy density for laminated grain oriented steel with $f=0.8$.
The corresponding relative permeabilities can be seen in Figure 6 (b). Because of the almost identical cross section compared to the full GO material, the in-plane curves $x$ and $y$ are also almost identical between (a) and (b). In contrast to that relative permeability in stacking direction $z$ is greatly reduced. With increasing saturation, the effect of the gap becomes less prominent, until both transverse directions are almost identical again. The contour surfaces or contour lines of the ferromagnetic material's contribution to the co-energy density $w_{\text {an }}^{\prime}$ according to (17) are almost indistinguishable from the co-energy density of the pure GO steel without lamination in Figure 7 and are therefore not separately shown. This is again because of the almost identical cross section.

The effect extends to higher co-energy densities with the lower stacking factor $f=0.8$ in Figure 9. The field in $z$ is dominated by the insulation even for higher fields where the ferromagnetic materials begins to reach the saturation. With the reduced cross section area of the ferromagnetic material, the flux in $x$ and $y$ is also slightly reduced and the saturation is reached a bit earlier.

Another example to test our model are magnetic shape memory alloys (MSMA). Figure 1 shows the characteristic HB-curves for the easy and hard axis. The results of the elliptic model are depicted in Figure 10. While the initial permeability of the GO steel was around 40000 in rolling direction, the MSMA only reaches 30. Therefore, higher co-energy densities are evaluated to cover the same flux density range. The MSMA's anisotropy is larger than that of the GO steel. The change in permeability isn't as high, consequently the contour lines in 2D are more equally spaced.
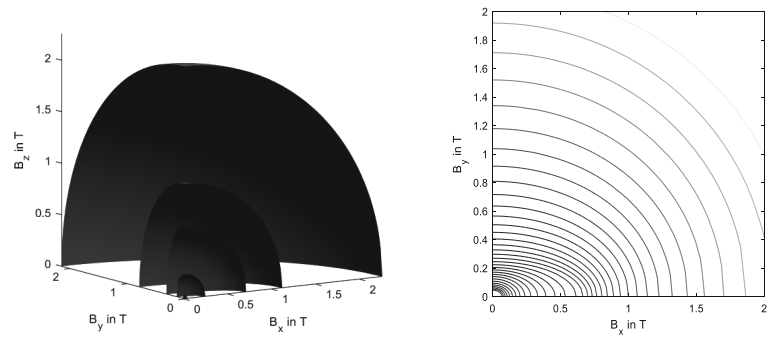

Figure 10: Contour lines of the magnetic co-energy density for MSMA with easy axis in $x$. 


\section{Numerical Validation}

To validate the laminated model, a benchmark FEsimulation is conducted in COMSOL, comparing the geometrically modelled laminate with an averaged continuum using the generated look-up table (9) at different angles with $\varphi=0^{\circ}$ denoting the flux in stacking direction $z$ and $\varphi=9^{\circ}$ ! for the flux in rolling direction $x$. For a decent accuracy, a table with 200 flux densities in each direction is used. As described in the introduction, magnetically anisotropic materials have to be implemented as relative permeabilities dependent on the flux density B. In contrast to that, the materials from the included material library are implemented as isotropic BH-curves as shown in Figure 5. To exclude errors in the developed anisotropic model, the comparison is therefore conducted with the isotropic material of GO M-6 in rolling direction from the same diagram. For the laminate, a stacking factor $f=0.95$ is investigated.

Additionally the relative permeability of the laminate rotated by $\varphi$ is calculated analytically. The co-energy density $w_{\text {an }}^{\prime}$ in the ferromagnetic material is

$$
w_{\mathrm{an}}^{\prime}(B, \varphi)=w_{\mathrm{an}}^{\prime}(B \sin (\varphi), 0, B \cos (\varphi)) .
$$

Using the look-up table, the relative permeabilities in material coordinates $\mu_{\mathrm{r}, i, \text { mat }}$ can be determined. Using those, the magnetic fields in material coordinates are

$$
\begin{aligned}
H_{x, \text { mat }}(B, \varphi) & =\frac{B \sin (\varphi)}{\mu_{\mathrm{r}, x, \text { mat }}\left(w_{\mathrm{an}}^{\prime}(B, \varphi)\right) \cdot \mu_{0}} \\
H_{z, \text { mat }}(B, \varphi) & =\frac{B \cos (\varphi)}{\mu_{\mathrm{r}, z, \text { mat }}\left(w_{\mathrm{an}}^{\prime}(B, \varphi)\right) \cdot \mu_{0}}
\end{aligned}
$$

The analytical field in flux direction and the relative permeability are then

$$
\begin{aligned}
H_{\mathrm{ana}}(B, \varphi) & =\sin (\varphi) H_{x, \text { mat }}+\cos (\varphi) H_{z, \text { mat }} \\
\mu_{\mathrm{r}, \text { ana }}(B, \varphi) & =\frac{B}{H_{\mathrm{ana}} \cdot \mu_{0}} .
\end{aligned}
$$

The model setup in COMSOL is shown in Figure 11. It consists of four tests, evaluated in the domain between the orange lines and connected parallelly to the same excitation. On the left, a material using the look-uptable of the laminated material is defined (magenta). To measure the material response at different angles, the material coordinates are rotated accordingly. This test of the averaged lamination model is designated "avg". The other three tests are different implementations of the

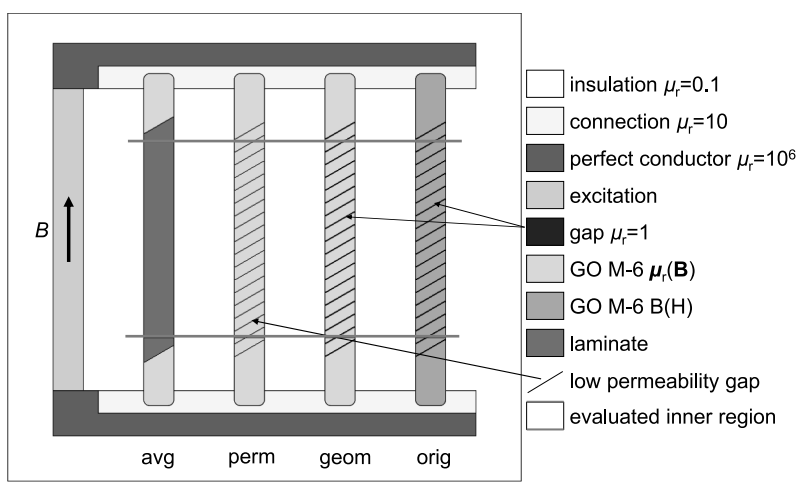

Figure 11: Model setup for rotation angle $\varphi=30^{\circ}$ with used materials and test designation at the bottom.

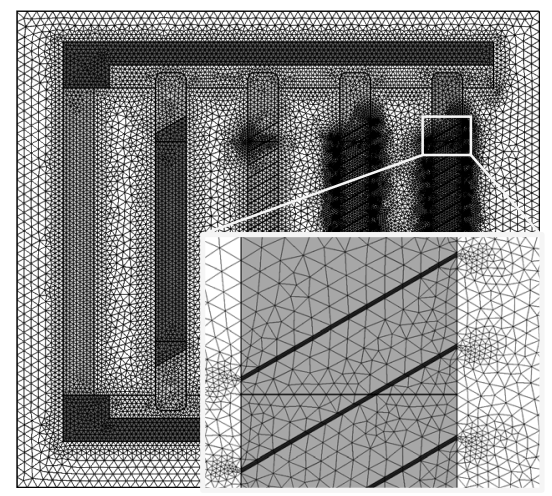

Figure 12: Used mesh, detail of the fully modelled laminate with two elements over the gap.

complete stacked geometry, which is again rotated for different cases.

The test designated "perm" uses a so-called "low permeability gap" between the layers of GO M-6. This special transition condition is used to model a thin gap of non-ferromagnetic material on a boundary instead of in a domain. An advantage is the easier mesh without the thin domains, the internal behaviour is not automatically calculated though. While the magnetic flux is computed correctly, the field strength and energy in the gap have to be considered manually to calculate the average permeability of the laminate.

The last two tests designated "geom" and "orig" use the fully modelled gaps. This can be seen in the detail of Figure 12, where the thin blue gap with $\mu_{\mathrm{r}}=1$ is shown. The gaps are identical for both tests, but the isotropic GO material is modelled differently. While for "geom" the look-up table is used, "orig" is modelled direly using the aforementioned BH-curve from Figure 5. 

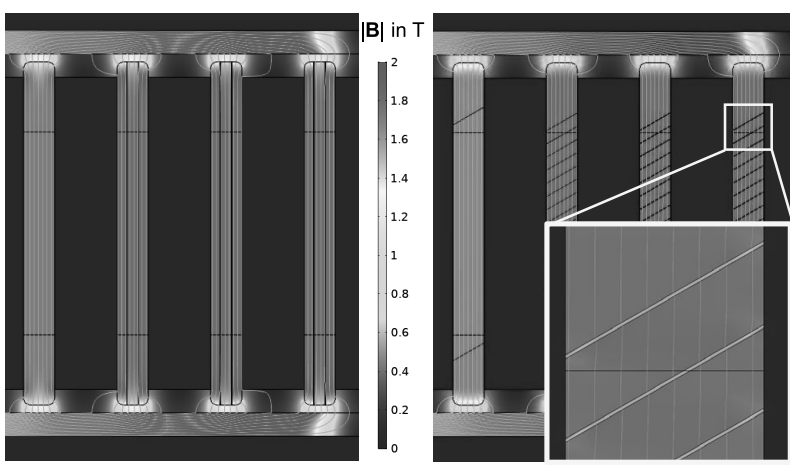

Figure 13: Resulting fields for an excitation of 7T, orientation of the laminate left $\varphi=90^{\circ}$, right $\varphi=3^{\circ}$ !, fluxlines in light grey.

The excitation has to be connected to the angled tests in a numerically stable way for a wide flux range. This is done in three steps. First, a vertical remanent flux is defined in the green excitation domain, this flux is distributed through the red almost perfect conductor with $\mu_{\mathrm{r}}=10^{6}$. To avoid steep gradients at the interface to the laminated material especially at $\varphi=90^{\circ}$ (see Figure 13 on the left), a connection layer with a lower permeability of $\mu_{\mathrm{r}}=10$ is defined and the corners are rounded. After a short layer of the non-laminated GO material, the test materials start outside the evaluated inner region. The necessary air region around the flux guiding geometries has a reduced permeability of $\mu_{\mathrm{r}}=0.1$. While this magnetic insulation is unphysical, it helps to concentrate the flux inside the test domains even at high saturation. Without this addition, the flux inside the test domains would be less uniformly vertical. We can therefore be even more accurate at high flux densities than physical experiments with the same geometry could be. The mesh can be seen in Figure 12. While a sufficiently fine mesh is defined for most domains, special care is required at the thin gaps in "geom" and "orig". These are done with a mapped mesh with two elements over the gap as shown in the detail view.

The local flux densities for two load cases can be seen in Figure 13. Both show an excitation of 7T which results in approximately $1.8 \mathrm{~T}$ in the test domains. Therefore, the GO steel is already in the beginning saturation according to Figure 6. While the overall flux is oriented vertically in the test domains in all cases, the detail at $\varphi=30^{\circ}$ shows a zig-zag pattern of the (grey) flux lines between the GO regions and the gaps. Therefore, the bridged length of the gap is reduced and accordingly

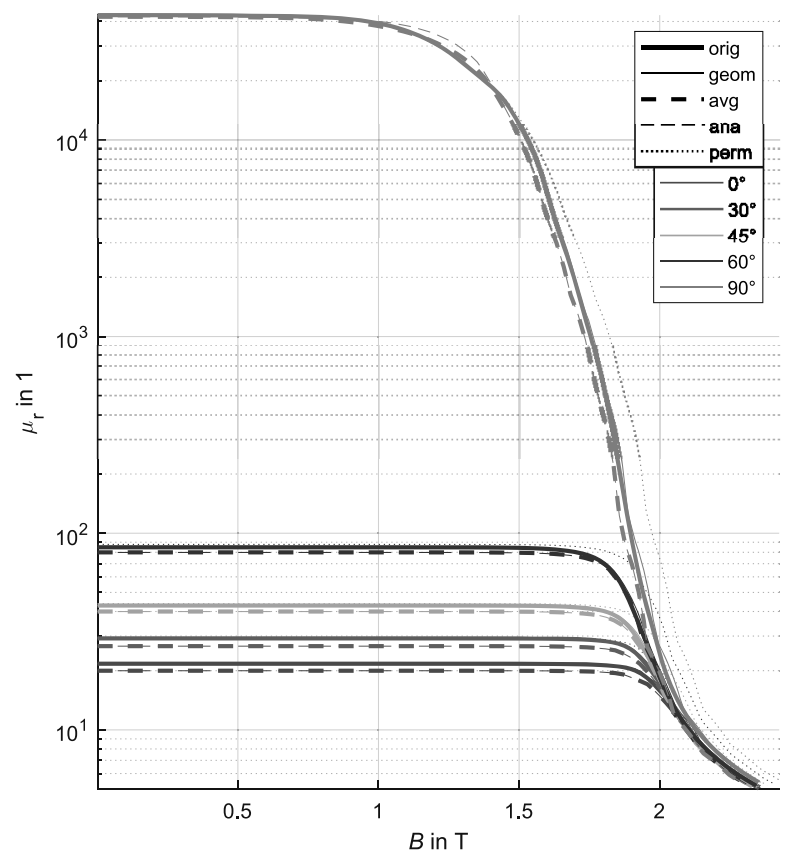

Figure 14: Resulting relative permeability $\mu_{\mathrm{r}}(B)$ for the specified tests (line styles) and angles (line colours).

the magnetic resistance. A much thinner, almost onedimensional geometry, would reduce this effect. In turn a sleek test region would suffer more flux leakage through the adjacent air regions.

The reduced magnetic resistance of the geometrically modelled tests "geom" and "orig" for diagonal layers compared to the averaged model can be also seen in the resulting relative permeabilities in Figure 14. There, the angles are colour coded and the four test scenarios and the analytic calculation according to (20) are denoted with different line styles. The two geometrically modelled tests "geom" and "orig" are shown with full lines, with a thicker line for "orig", the implementation using the original material model. Apart from some ripples in the saturation region a complete fit can be observed. Those ripples are caused by the interpolation on the look-up table and dependent on its resolution.

The simulated averaged model is marked with a thick dashed line. The thin dashed line denotes the test "ana". This is the analytical calculation according to (20) applied to the average flux density in the test domain. Apart from a slight deviation at the beginning saturation at $\varphi=90^{\circ}$, the analytical calculation "ana" and the implemented model "avg" are a complete match. The ripples in both curves are again caused by the resolution of the 
look-up table. The dotted line denoting the test "perm" with the low permeability gap overestimates the permeability at higher flux densities in rolling direction (higher angles towards $\varphi=90^{\circ}$ ), because the reduced cross section of the ferromagnetic material cannot be taken into account in this setup. This deviation would increase for higher stacking factors.

The overall performance of the developed averaged model can be evaluated comparing the thick solid line for the original model and the thick dashed line for the averaged model. The saturation behaviour is replicated accurately for all angles. The starting permeabilities meet exactly the analytical expectation, while the original model slightly overestimates them for a flux in stacking direction (lower angles towards $\varphi=0^{\circ}$ ), most likely caused by the discretization in the thin gaps.

\section{Conclusion}

The proposed elliptic interpolation of the co-energy density produces convex functions for a stable and thermodynamically consistent simulation. The generated look-up table of the relative permeabilities $\mu_{\mathrm{r}, i}$ can be easily imported in FE-software like COMSOL Multiphysics. While we showed the interpolation between perpendicular uniaxial measurements, the data of other directions could be also included in the construction of the contour surfaces. This might be particular useful for GO steel at low fields where the angle between the magnetic hard direction and the easy rolling direction is close to $60^{\circ}$ as discussed in [9]. Our model can be applied to anisotropic materials with totally different permeability ranges as shown for GO steel and MSMA.

For laminated materials, with an increasing stacking factor the lamination becomes dominant compared to the anisotropy of the base ferromagnetic material. The overall behaviour of the laminate is dependent on the complete co-energy density $w_{\text {lam }}^{\prime}$, in contrast to that, the permeability is dependent on the co-energy density $w_{\text {an }}^{\prime}$ in the ferromagnetic material. Even very high stacking factors $f$ with only small gaps in the ferromagnetic material already cause a drastic change in the contour lines and surfaces of $w_{\text {lam }}^{\prime}$ compared to the material without lamination, while $w_{\text {an }}^{\prime}$ is less effected. The permeabilities calculated from $w_{\text {an }}^{\prime}$ are much smoother than those generated from the gradient of $w_{\text {lam }}^{\prime}$ and correctly replicate the high anisotropy.

The numerical validation shows a very high accordance of the fully modelled laminate and the deduced homogenization rule. This opens many possibilities for efficient models of magnetic circuits without the need to mesh the thin gaps. For dynamic simulations, the described magnetic behaviour is not sufficient. Besides hysteresis, the electrical conductivity $\sigma$ becomes important to estimate eddy currents. For laminated stacks, different models of $\sigma$ are compared in[10].

\section{References}

[1] S. Lopez, et al. Grain Oriented Steel Assembly Characterization for the Development of High Efficiency AC Rotating Electrical Machines, IEEE Trans Magn. 2009; 45(10): 4161-4164. doi: 10.1109/TMAG.2009.2023243

[2] Y. Ushigami, et al. Recent development of low-loss grain-oriented silicon steel, J Magn Magn Mater. 2003; 254-255: 307-314. doi: 10.1016/S0304-8853(02)00933-2

[3] T. Nakata, et al. Measurement of magnetic characteristics along arbitrary directions of grain-oriented silicon steel up to high flux densities, IEEE Trans Magn. 1993; 29(6): 3544-3546. doi: $10.1109 / 20.281224$

[4] D. Miyagi, et al. Development of Measuring Equipment of DC-Biased Magnetic Properties Using Open-Type Single-Sheet Tester, IEEE Trans Magn. 2006; 42(10): 2846-2848. doi: 10.1109/TMAG.2006.879144

[5] A. Di Napoli, R. Paggi. A model of anisotropic grain-oriented steel, IEEE Trans Magn. 1983; 19(4): 1557-1561. doi: 10.1109/TMAG.1983.1062582

[6] J.C. Sabonnadiere, G. Meunier. Modelling Nonlinear Magnetic Materials for Field Computation, J ELECTROMAGNET WAVE. 1990; 4(11): 1027-1054. doi: 10.1163/156939390X00357

[7] J. Krause, Energy conserving Anisotropic Anhysteretic Magnetic Modelling for Finite Element Analysis. 2012; arXiv:1212.5163v1 [math-ph]

[8] M. Schautzgy, U. Kosiedowski, T. Schiepp. 3D-FEM-Simulation of Magnetic Shape Memory Actuators, Proc. of 2016 COMSOL Conf.; 2016 Oct; Munich.

[9] H. Wang, C.-S. Li, T. Zhu. Hard magnetization direction and its relation with magnetic permeability of highly grain-oriented electrical steel, INT J MIN MET MATER. 2014; 21(11): 1077-1082. doi: $10.1007 / \mathrm{s} 12613-014-1012-8$

[10] J. Ziske, H. Neubert R. Disselnkötter. Modeling of Anisotropic Laminated Magnetic Cores using Homogenization Approaches, Proc. of 2014 COMSOL Conf.; 2014 Sep; Cambridge. 